\title{
Carbon Nanotubes as Optoelectronic Energy Transducers
}

\author{
Eyal Shafran,* Benjamin D. Mangum, ${ }^{* *}$ and Jordan M. Gerton*
}

* Department of Physics and Astronomy, University of Utah, 115 South 1400 East, Salt Lake City, UT 84112

** Current Address: Soft Matter Nanotechnology and Advanced Spectroscopy Team, Los Alamos National Laboratory, Los Alamos, NM 87545

The transduction of energy from one type to another is frequently a limiting factor in the efficiency of optoelectronic devices. Such transduction processes are often necessary for energy to propagate across the interface between materials. As an example, next generation solar cells may require several energy transduction steps: conversion of electromagnetic radiation to internal energy of one component, to internal energy of another component, and finally to electric power. In this scenario, each sequential transduction process must be more rapid than the internal relaxation time, or the energy will be dissipated as radiation or heat. Recently, we demonstrated that when a carbon nanotube (CNT) is brought into close proximity with a fluorophore, the fluorophore's emission is nearly completely quenched $[1,2]$. This indicates that the fluorophore's internal energy is transduced into electronic modes within the CNT. Nanotubes are particularly intriguing candidates for studying nanoscale energy transduction because on the one hand, they can support ballistic charge transport, and on the other, the detailed electronic properties vary from tube to tube according to its chirality. Furthermore, they have been synthesized as dense vertically-aligned networks, facilitating their incorporation into complex optoelectronic materials, and finally they have been used as nanoscale components in molecular electronics.

Carbon nanotubes (CNTs) have been proposed as a charge-transport element in next-generation optoelectronic devices and materials, including photovoltaics [3]. Indeed, nano-composite materials with a strong light absorption component (i.e., fluorophores) coupled to a CNT component have been synthesized via several different methods. The interfacial area between donor and acceptor in a nanocomposite material is extremely large, so nanoscale energy transfer is very important, but difficult to measure in a controlled and precise manner. We used individual CNTs attached to atomic force microscope (AFM) tips to probe isolated quantum dots (QDs) while measuring the fluorescence signal. Figure 1(a) shows an example of a fluorescence approach curve; the far-fieldnormalized fluorescence signal, $S(z)$, is plotted as a function of the vertical separation, $z$, between the CNT terminus and QD surface; also shown is an $x-z$ tomographical section [4] of a QD. The strong reduction in fluorescence was observed consistently in more than 100 measurements on $>50$ QDs using 6 different CNTs [1]. All data agree to high precision with energy transfer via a Förster dipoledipole coupling between a photoexcited exciton in the QD and a resonantly-excited exciton in the CNT. However, this agreement is only achieved if the standard Förster model is modified to account for the possibility of creating an exciton above the CNT terminus. This model predicts a strong correlation between the Förster radius and the average position at which the exciton is created within the CNT, in agreement with measurements. The model predicts the peak energy transfer efficiency 
should saturate at a value of $\sim 0.96$, which again agrees with measurements, as shown in Fig. 1(c). This saturation seems to be independent of whether the CNT is semiconducting or metallic.

For some QDs, the peak energy transfer efficiency of $\sim 0.96$ is maintained even when the energy transfer must compete with faster internal relaxation of the QD (i.e., lower quantum yield). This implies that the energy transfer rate also increases to compensate for more rapid internal relaxation. In contrast, some QDs are not as robust, and the peak energy transfer efficiency decreases when the intrinsic relaxation processes become faster. Thus, it seems there are (at least) two classes of QD that yield significant differences in energy transduction efficiency. This issue can be studied by utilizing the fast cycling between different internal states (with different quantum yield values) of a QD that occurs during "blinking" [2]. In particular, we developed a novel single-photon counting technique [4] that allows each detected photon to be correlated with both the instantaneous height of the CNT above the QD and also the intrinsic quantum yield of the QD at that instant. Thus, from a single measurement on a blinking QD, it is possible to produce many different QD-CNT interaction curves corresponding to different quantum yield values. This method has already been used to study how the near-field interactions between an emitter and a nearby nanostructure (i.e., AFM tip or $\mathrm{CNT}$ ) can be tuned via the quantum yield [2]. These results are very fundamental in nature, but they have implications for composite materials composed of QDs attached to CNTs, which are being developed for a variety of optoelectronic applications, including photovoltaics and sensing.

\section{References}

[1] E. Shafran, B.D. Mangum, and J.M. Gerton, Nano Lett. 10 (2010) 4049.

[2] E. Shafran, B.D. Mangum, and J.M. Gerton, arXiv 1102.2962v1 (2011).

[3] V. Biju, T. Itoh, Y. Baba, and M.J. Ishikawa, Phys. Chem. B 110 (2006) 26068.

[4] B.D. Mangum, E. Shafran, C. Mu, and J.M. Gerton, Nano Lett. 9 (2009) 3440.

[5] This work was supported by a Cottrell Scholar Award from the Research Corporation for Science Advancement and by an NSF CAREER Award number DBI-0845193.
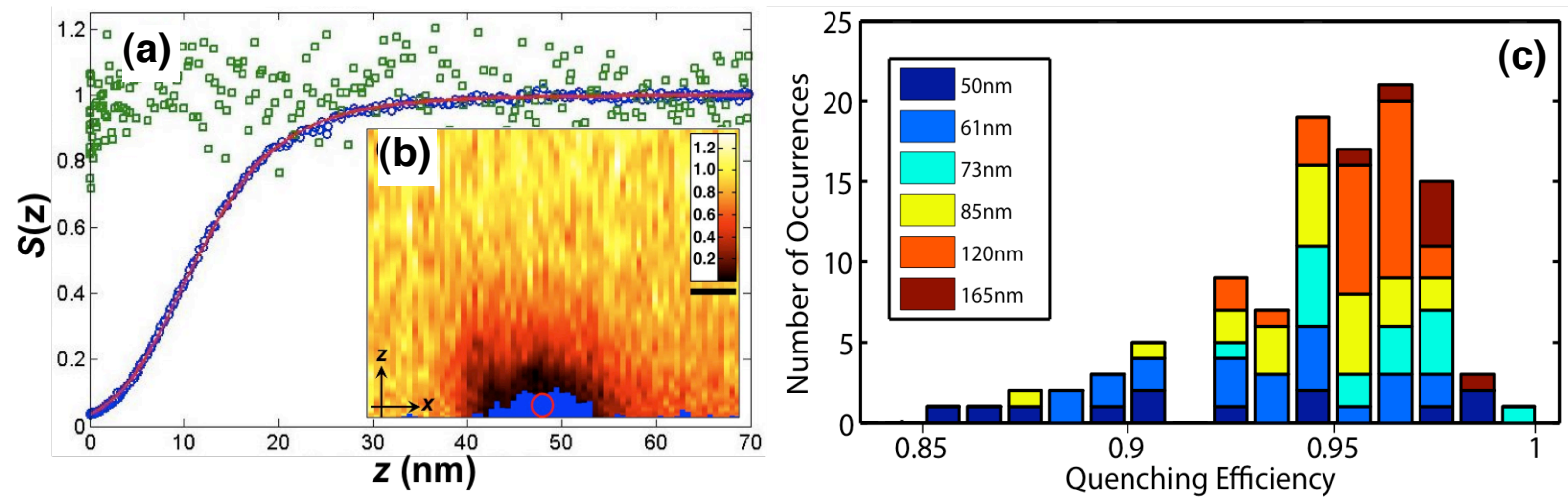

FIG. 1. Energy transfer between a QD and CNT. (a) Approach curve for the bright state of a QD (blue symbols); the solid red line is a fit to the Förster model. (b) $x-z$ tomographical section. The blue cutout region denotes the AFM height trace and the color scale gives the normalized fluorescence signal. The scale bar is $10 \mathrm{~nm}$. (c) Histogram of the peak energy transfer efficiencies at QD-CNT contact. The data are color-coded according to the length of each CNT used. 